\title{
Focus Energy Determination of Mining Microseisms Using Residual Seismic Wave Attenuation in Deep Coal Mining
}

\author{
Mingwei Zhang $\mathbb{D}^{1,2}$ Shengdong Liu $\mathbb{D}^{1,},{ }^{1,2}$ Shuzhao Chen, ${ }^{3}$ Yanlong Chen $\mathbb{D}^{1,2}$ \\ Guang Xu $\oplus^{4},{ }^{4}$ and Deyu Qian $\mathbb{1}^{3}$ \\ ${ }^{1}$ State Key Laboratory for Geomechanics and Deep Underground Engineering, China University of Mining and Technology, \\ Xuzhou, Jiangsu 221116, China \\ ${ }^{2}$ School of Mechanics and Civil Engineering, China University of Mining and Technology, Xuzhou, Jiangsu 221116, China \\ ${ }^{3}$ School of Mines, China University of Mining and Technology, Xuzhou, Jiangsu 221116, China \\ ${ }^{4}$ Department of Mining Engineering \& Metallurgical Engineering, Western Australian School of Mines, Curtin University, \\ Kalgoorlie, WA 6430, Australia
}

Correspondence should be addressed to Mingwei Zhang; mingweizhang@cumt.edu.cn and Shengdong Liu; vipshengdongliu@sina.com

Received 20 October 2017; Accepted 26 December 2017; Published 21 February 2018

Academic Editor: Longjun Dong

Copyright (C) 2018 Mingwei Zhang et al. This is an open access article distributed under the Creative Commons Attribution License, which permits unrestricted use, distribution, and reproduction in any medium, provided the original work is properly cited.

\begin{abstract}
Based on the energy attenuation characteristics of residual wave in deep rock, a method was developed to determine the microseismic focus energy. Differential energy loss in infinitesimal spreading distance is logically deduced, upon which energy attenuation equation was established. With a logarithmic transformation, a linear relation of the residual seismic energy with distance is formulated. Its intercept was used to determine the microseismic focus energy. The result is compared with that determined by the energy density method. The reliability of the determined focus energy and the impact of the built-in velocity threshold on the residual wave energy computation are discussed. Meanwhile, the energy absorption coefficient used for representing the absorption characteristics of the rock medium in the mining region under study is also clarified. Key findings show that the microseismic focus energy confirmed by the residual wave attenuation is reliable. The result's accuracy is quite high, especially for the events in deep rock with great homogeneity. The developed focus energy computation method is closely dependent on the integrity of waveform, accuracy of repositioning, and reliability of effective components extraction. The new method has been shown to be effective and practical.
\end{abstract}

\section{Introduction}

Due to the large-scale exploitation in recent years, shallow coal resources gradually decrease and the mining depth increases year by year [1]. Underground geological dynamic disasters such as rock burst and coal and gas outburst are getting more and more serious with the increase of mining depth and exert a negative influence on safe mining [1-3]. To eliminate the adverse impact, microseismic technique developed from mining geophysics is applied gradually into the monitoring, assessment, prevention, and control of dynamic disasters in these years and has already played a certain effective role [4-8]. For the passive microseismic method, the focus position and releasing energy of microseismic event are two significant fundamental elements to ensure the availability of a correlative solution. Microseismic focus position and energy indicate the mode, range, and direction of rock failure and stress drop in a specific spatial scale, which prompts microseismic method to become a principal advanced approach for dynamic disaster control and helps to achieve the great progress in mining hazard management [9-11]. As such, accuracy and reliability of focus position and focus energy become one of the main research interests and are already in full swing [11-13]. The former started earlier and is more mature. By contrast, determination of elastic strain energy released from microseismic focus has been paid less attention. Current focus energy computation methods applied for microseisms such as the seismic duration 


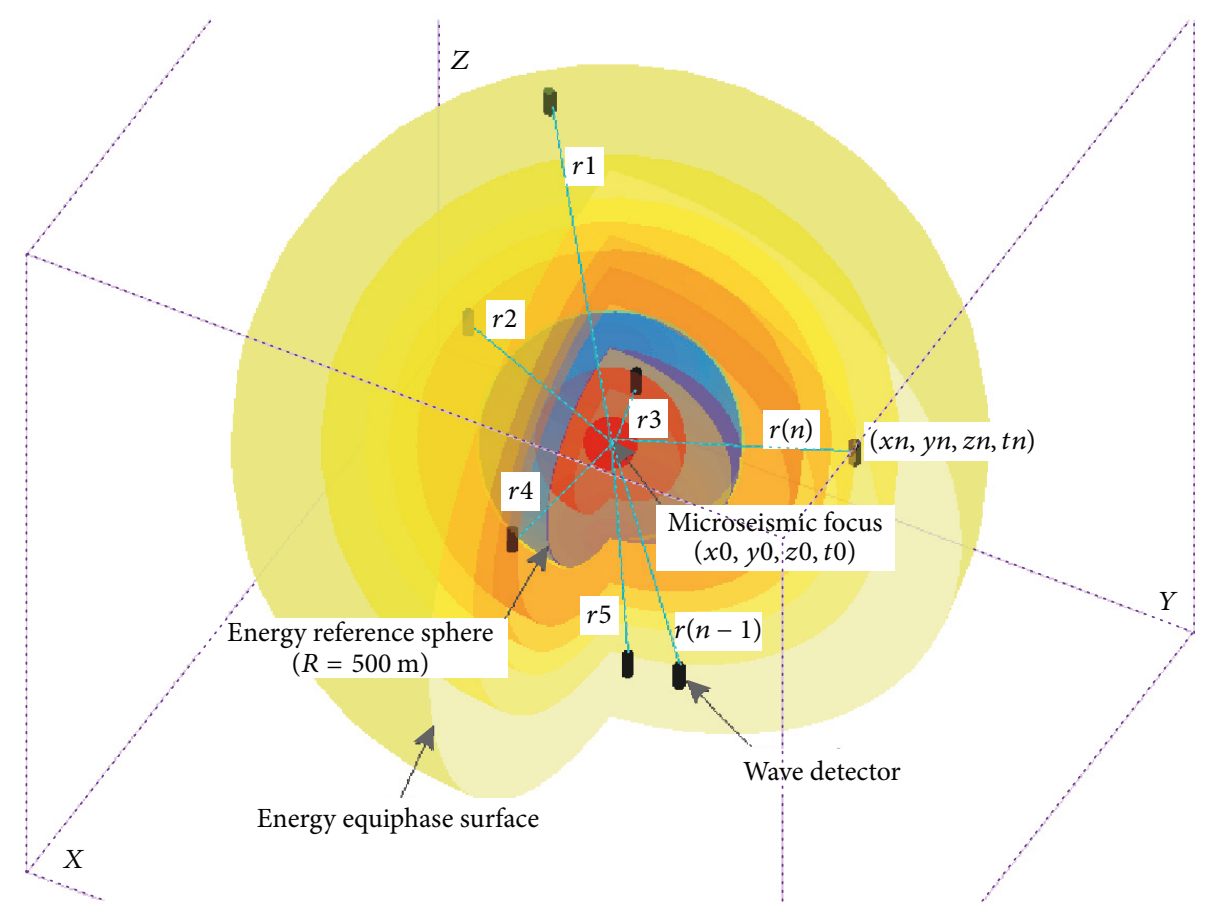

FIGURE 1: Energy emission of microseismic event in deep rock and the reference sphere model for focus energy calculation.

and phase integral are mainly referred from the seismology, for which equivalent energy density using the reference equiphase surface is the common approach [14-19].

However, it is commonly found that microseismic focus energy obtained from the energy density method is relatively defective and is not reliable enough $[20,21]$. As obtained from the idealized theoretical model, focus energy computation is easily influenced by geological difference, manual operation, and firmware performance $[22,23]$. Relative dispersion of individual focus energy obtained from different channels is great with the high dependency on the physical properties of rock medium. And a $10^{2} \sim 10^{5}$ times of difference exists between the maximum and the minimum individual energy magnitude. The reliability of final focus energy determined by the mathematical average is low, especially in complicated geological conditions. In addition, focus energy variation results from artificial influences $[24,25]$. For the same microseismic event, diverse focus energy magnitude is processed obviously by different operators. Disparate focus energy magnitude is also obtained from repetitive solutions obtained by the same operator. This makes calculated results of focus energy have an obvious uncertainty and great randomness.

Microseismic focus energy deviation does not help for the dynamic disaster management in deep mining [2, 26]. Accurate focus energy contributes to positive hazard management [27]. Evaluation on geological dynamic disasters shall get more scientific and effective once conducted upon the more reliable focus energy. Thus, in this study, based on the exploration on energy attenuation characteristic of microseismic wave in deep rock, the authors put forward a more objective method for computing microseismic focus energy.

\section{Attenuation Characteristics}

After a microseismic event occurs, a large amount of elastic strain energy releases rapidly and propagates in surrounding rock mass in the form of stress wave. An idealized propagation of the spherical radiant energy released from the microseismic focus is demonstrated in Figure 1. During the wave propagation in deep rock, wave energy attenuates gradually with the increase of propagation distance. The impact of rock mass on the microseismic wave energy attenuation is significant. Energy attenuation behavior is closely related to the physical properties and structures of rock mass $[28,29]$. Energy attenuation effect is induced from the scattering conversion and absorption action of the propagation medium, and energy absorption is the dominant intrinsic attribute of rock medium. Wave energy attenuation contains information about microscopic properties and physical changes of rock structure such as density and distribution of internal cracks, which is fundamentally important to describe deep rock [30, 31].

Energy attenuation behavior of microseismic wave has some apparent characteristics such as the rapid decrease of peak particle velocity (PPV) and amplitude with the increasing propagation distance from focus [32]. Decay of the PPV and wave amplitude is synchronized, which directly reflects the intensity of a microseismic event. Energy attenuation behavior also appears in the frequency spectrum. An attenuation case of microseismic wave energy in the entire frequency spectrum is shown in Figure 2. Microseismic wave components in the entire frequency domain are absorbed by the rock medium. Compared with the energy attenuation in low frequency range, dissipation of wave components in high 


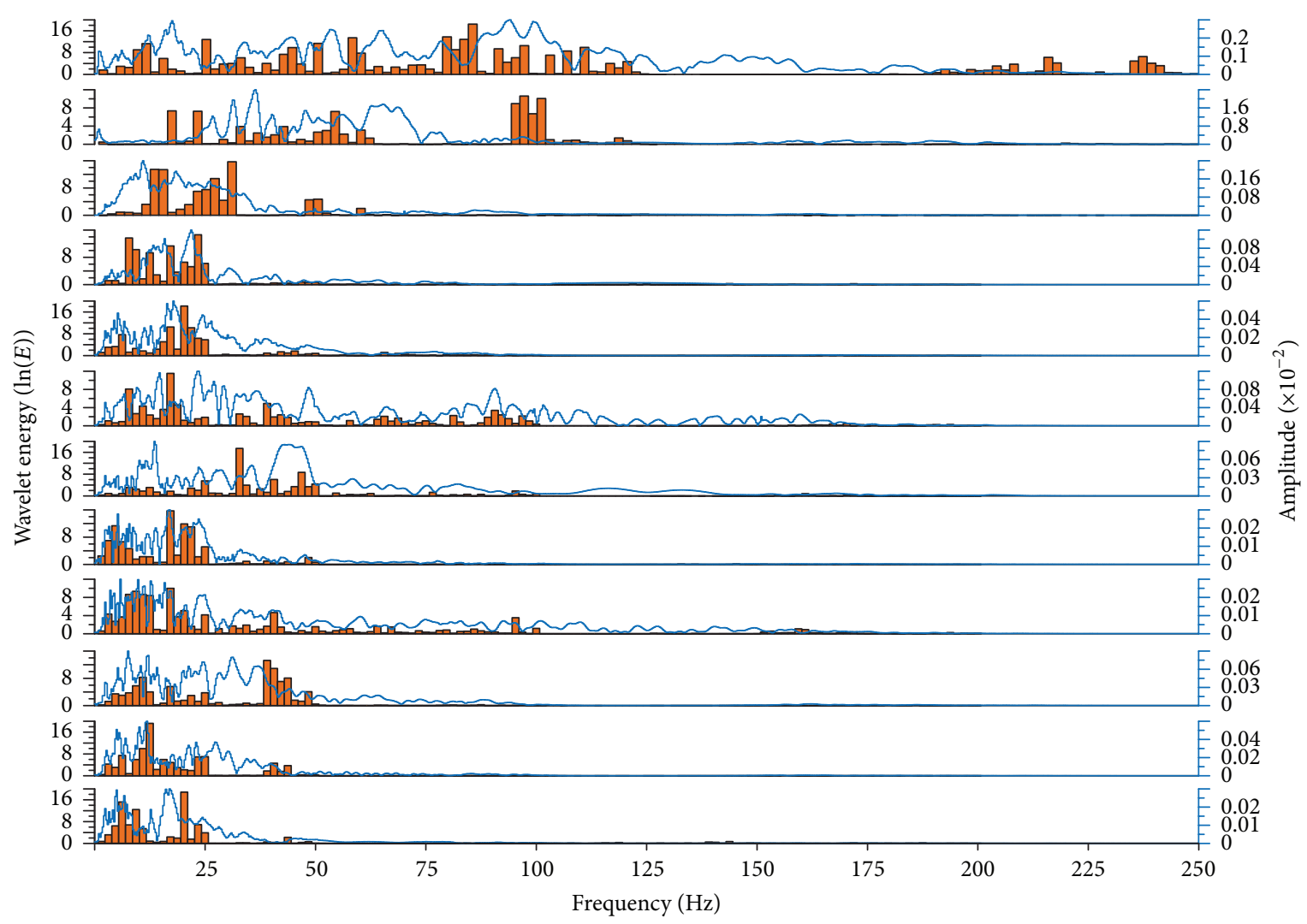

Figure 2: Congruent relation of frequency spectrum and energy distribution of microseismic wave during the remote attenuation.

frequency range is more obvious and complete. The width and amplitude of the dominant frequency band decrease gradually, which leads most of the residual high-energy wave components to concentrate in the low frequency range [33]. This filtering action of propagation medium increases the wave duration, yet it decreases the wave resolution. Compared to the wave with a short propagation distance, remote microseismic wave shows up the briefer features in frequency-energy spectrum.

If the wave energy $E$ is residual as the propagation distance is $R$, a differential energy $d E$ will be lost in the subsequent infinitesimal spreading distance $d R$ as shown in Figure 3. This is expressed in the following equation:

$$
-d E=2 \alpha E d R .
$$

Then, the energy attenuation behavior of microseismic event in the propagation medium is defined as

$$
E=E_{0} e^{-2 \alpha R}
$$

where $E_{0}$ is the original focus energy, $R$ is the wave propagation distance, $E$ is the residual energy at a specific location, and $\alpha$ is the energy absorption coefficient. This quantificational relation reveals that the elastic energy released from a seismic event dissipates exponentially with the increase of propagation distance. Meanwhile, energy absorption coefficient is an important parameter representing the dissipation

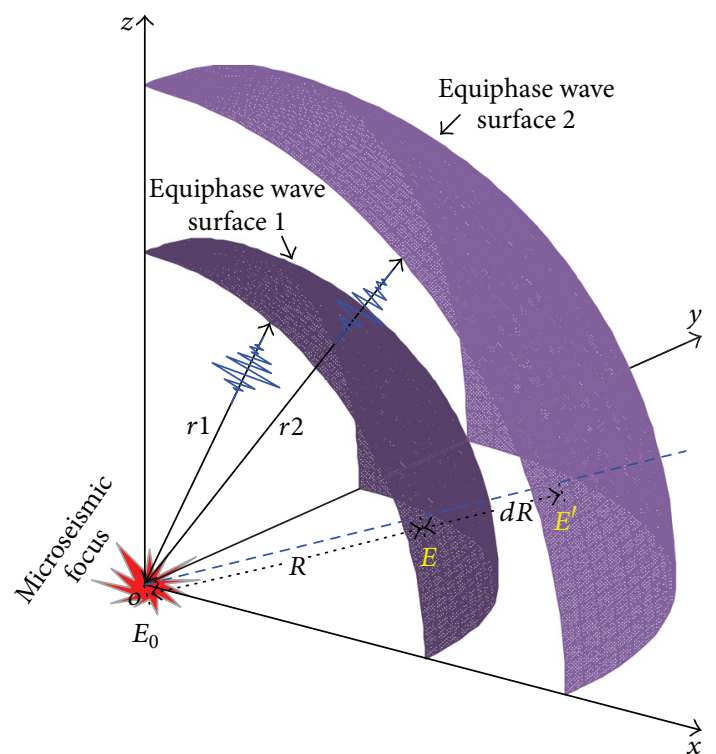

FIGURE 3: Theoretical analysis model of microseismic focus energy attenuation behavior.

characteristics of wave energy in rock mass and plays a primordial role in mirroring the vibration behavior, inelastic performance, and intrinsic property of propagation medium. 


\section{Reverse Analytical Thought for Microseismic Focus Energy Determination}

Equation (1) is applied for solving the residual elastic energy at a specific location. It indicates the definite relationship between the initial wave energy at microseismic focus and residual wave energy at somewhere with a specific propagation distance. To make the residual wave energy clear, energy absorption coefficient and microseismic focus energy are the two essential elements that shall be known beforehand. However, they are not available for passive microseismic event. It requires that the residual wave energy at a specific location shall be known firstly. There are four unknown variables in this equation. Only when the residual wave energy at a specific propagation location is known can the energy absorption coefficient and microseismic focus energy be possibly solved upon at least two equation sets. Essentially, based on the focus repositioning and wavelet packet transform [13, 34-36], the more accurate focus location, wave propagation distance, and residual wave energy are feasibly computed, which theoretically makes it practicable for a microseismic event to establish the mathematical relation between known variables and unknown variables and then to obtain the corresponding energy absorption coefficient and focus energy. Thus, the Napierian logarithmic transformation of focus energy attenuation equation is processed as follows:

$$
\ln (E)=\ln \left(E_{0} e^{-2 \alpha R}\right)
$$

then,

$$
\ln (E)=-2 \alpha R+\ln \left(E_{0}\right)
$$

The above equation reveals that Napierian logarithm of residual wave energy is linearly dependent on wave propagation distance, in which elements $-2 \alpha$ and $\ln \left(E_{0}\right)$ are the slope and intercept of the straight line, respectively. Under the preconditions of known wave propagation distance and residual wave energy, the slope and intercept of the straight line can be confirmed. If $k=-2 \alpha$ and $B=$ $\ln \left(E_{0}\right)$, the microseismic focus energy and the relevant energy absorption coefficient are naturally obtained by the following expressions:

$$
\begin{aligned}
E_{0} & =e^{B} \\
\alpha & =-\frac{k}{2} .
\end{aligned}
$$

Based on the attenuation characteristics of microseismic wave energy and the indispensable Napierian logarithmic transformation, not only is the magnitude of microseismic focus energy explored by the intercept $B$, but also the energy absorption coefficient can be determined by the slope $k$. Exponent $B$ is the key parameter that determines the amount of released elastic strain energy from the microseismic focus. It can be obtained by the fitted attenuation expression. This is the precise analytical thought for microseismic focus energy determination method recommended in this research.

\section{Results}

4.1. Field Investigation. To specify the theoretical relation, clarify the feasibility of the analysis thought, and further make the energy computation method more representative and universal, fifteen days of field investigation using the continuous microseismic monitoring operation were carried out in the deep Xingcun Coal Mine, located in East China. 10 detectors were located in underground coal mining region. In total, 92 available microseismic wave groups were randomly screened from the database and treated as the analysis objects. Original waveform of a microseismic event is shown in Figure 4. Generally, as the noise interference, original microseismic wave is unqualified to be directly applied into the wave energy computation, especially for the microseism with a remote propagation distance [37]. The denoising process using the effective wave component extraction in the time domain, frequency domain, and energy domain is necessary to decrease the noise, improve the signal-tonoise ratio, purify the waveforms, and highlight the wave substantive characteristics. After the effective component extraction, variation of the basic characteristic information of the microseismic event is revealed in Table 1. Here, arrival time was determined using the $\mathrm{P}$-wave.

\subsection{Relation Establishment of the Residual Wave Energy} versus Wave Propagation Distance. Corresponding to the reverse analytical thought for microseismic focus energy computation, quantitative relation of residual wave energy versus wave propagation distance is established as shown in Figure 5. In this case, all of the residual wave energy is logarithmically processed and wave propagation distance is confirmed upon the focus repositioning. This relation reveals the attenuation characteristics of the residual microseismic wave energy. Upon the least square method, linear relation of logarithmic wave energy and propagation distance is fitted as

$$
\ln (E)=-0.0057 R+9.9189 \text {. }
$$

Then, the magnitude of the energy absorption coefficient and the focus energy of the microseismic event is inversely obtained:

$$
\begin{aligned}
\alpha & =-\frac{-0.0057}{2}=2.85 \times 10^{-3} \\
E_{0} & =e^{9.9189}=20310.64 .
\end{aligned}
$$

The new microseismic focus energy of the individual case is 1.36 times greater than that obtained from the energy density method, which indicates that determination of microseismic focus energy is completely feasible on the basis of the wave energy attenuation characteristics.

Meanwhile, it is also noted in Figure 5 that the exponential fitting relation between the logarithmic residual wave energy and the wave propagation distance possesses greater correlation coefficient. After the logarithmic transformation, the exponential fitting reflects the quantitative relationship better and more sensitive than the linear fitting. However, this phenomenon does not mean that microseismic focus 


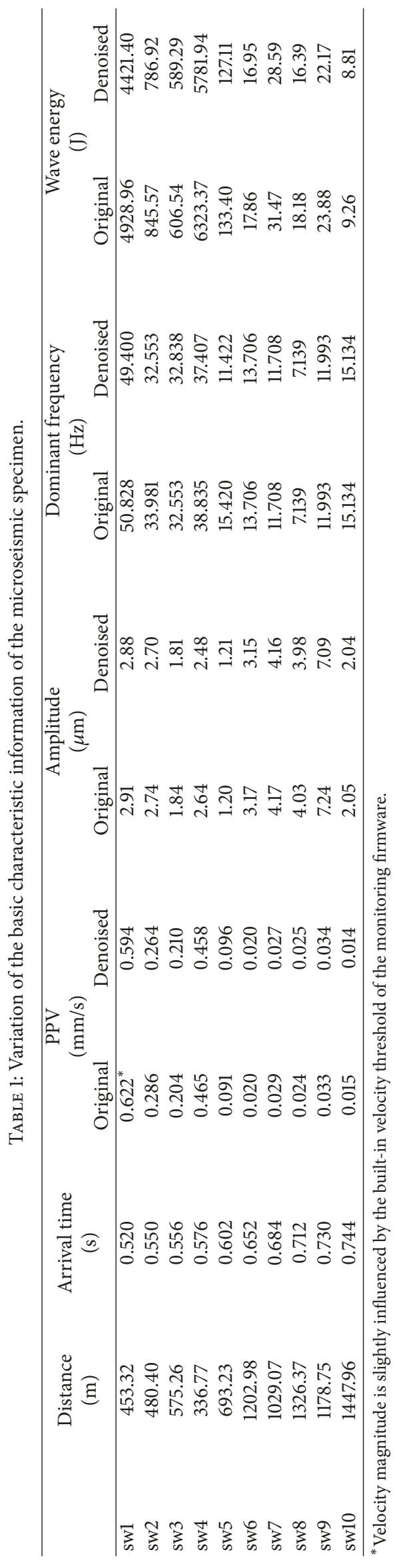




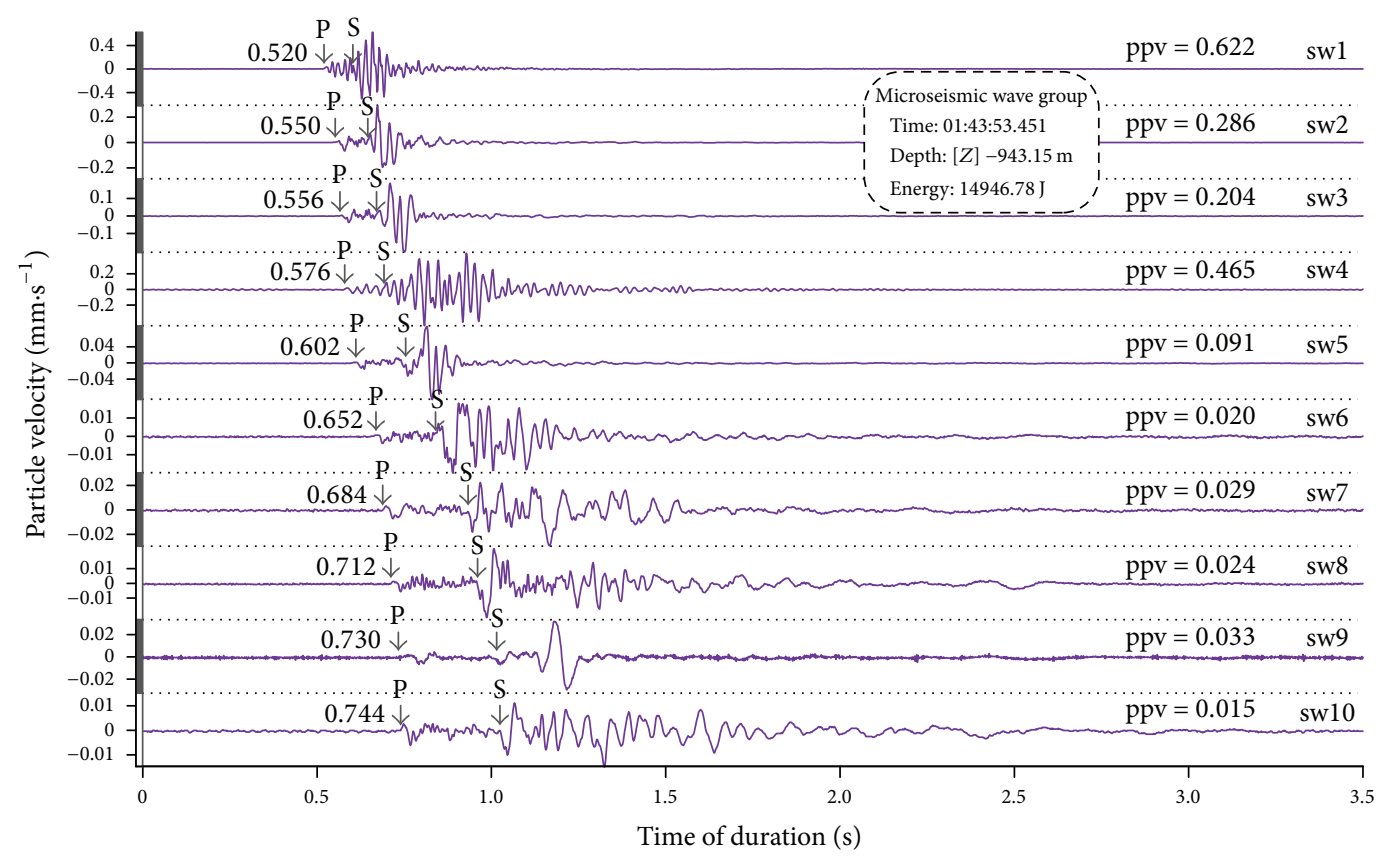

FIGURE 4: Original waveform of a microseismic event obtained in the field investigation.

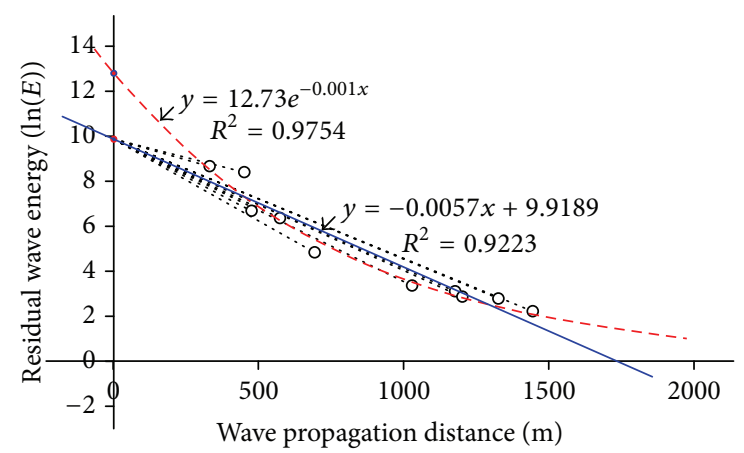

FIGURE 5: Attenuation of residual microseismic wave energy in coordination with the increasing propagation distance.

energy calculated using the exponential fitting relation is more accurate. The microseismic focus energy confirmed by the exponential fitting is

$$
E_{0}=e^{12.73}=337729.31 \text {. }
$$

This magnitude of focus energy is equivalent to a ML0.48 unfelt earthquake. In deep underground mining, this level of seismic effect is destructive, which is at variance with the reality. The exponential fitting relation is consistent with the rules of wave energy attenuation in classical seismology.

4.3. Big Data Analytics on the Targeted Verification of the Inverse Microseismic Focus Energy Determination. Similarly, for the other 91 microseismic events, the linear relations between the logarithmic residual wave energy and wave propagation distance are revealed in Figure 6 using the same treatment. The redetermined magnitude of the microseismic focus energy compared to the original ones is listed in Table 2.

Field experiment shows that, during the entire attenuation of microseismic wave in deep rock, variation of logarithmic residual wave energy and the corresponding propagation distance is consistent with the linear relation. The linear attenuation characteristics of logarithmic microseismic focus energy are fairly obvious at the near-focus location, for which the propagation distance is generally less than $500 \mathrm{~m}$. The energy attenuation gets conspicuous at the location that is closer to the focus.

\section{Discussions}

5.1. Critical Parameters and Resolution. Residual wave energy and wave propagation distance are the two critical fundamental factors for the new focus energy computation method. The quantitative relation can only be established in the mathematic fitting algorithm if the above factors are fixed. Residual wave energy is determined by the integrity of waveform and the reliability of effective wave components. No matter where a microseism is monitored and how much elastic energy the event releases, the monitored microseismic signal with a specific waveform is inevitably corresponding to a naturally given residual energy. The key point is just the determination of residual wave energy. All of the residual wave energy magnitude applied in this study is calculated from the microseismic wave reconstructed by the purified effective components extracted by the domain decomposition method. The wave propagation distance is confirmed by the focus repositioning. As the microseism is passive, its focus location is indeterminate. Better focus repositioning contributes to a more reliable logical relationship. The crucial 


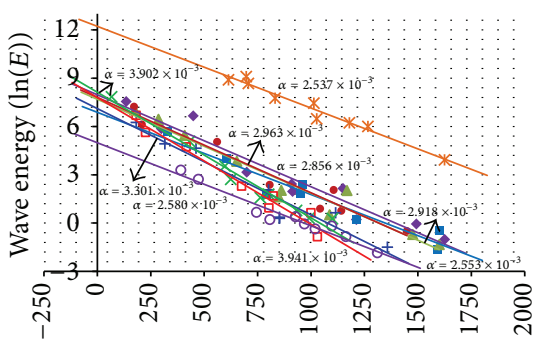

Wave propagation distance $(\mathrm{m})$

$$
\text { - D1-1 }
$$

- D1-2

+ D1-6

$\triangle \mathrm{D} 1-3$

$\times \mathrm{D} 1-7$

- D1-4

* D1-5

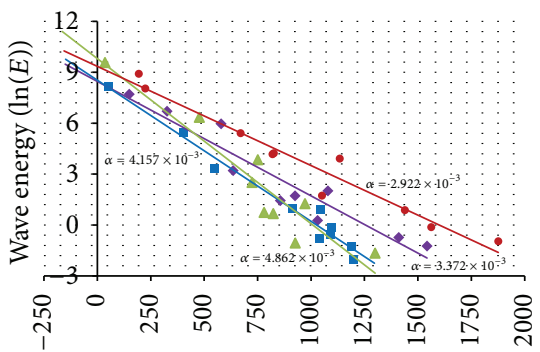

Wave propagation distance $(\mathrm{m})$

- D4-1

- D4-2

$\triangle \mathrm{D} 4-3$

- D4-4

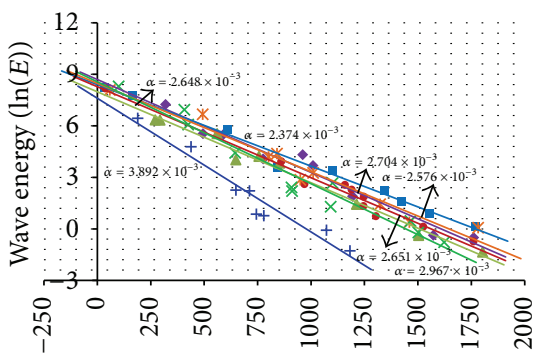

Wave propagation distance $(\mathrm{m})$
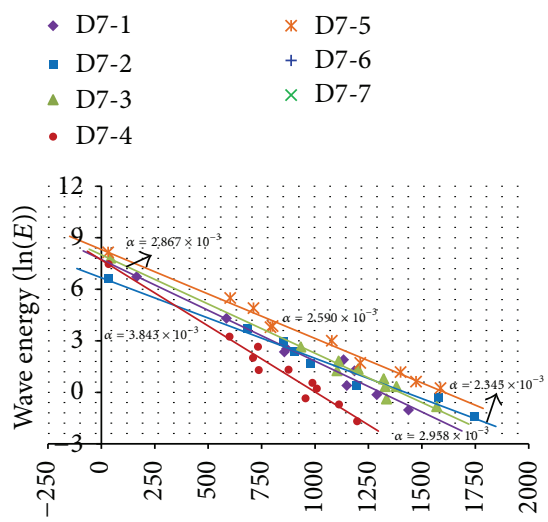

Wave propagation distance $(\mathrm{m})$

- D10-1

- D10-4

$\Delta$ D10-3

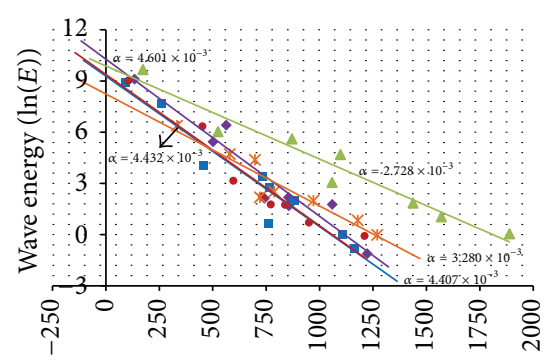

Wave propagation distance (m)

- D2-1

- D2-4

- D2-2

* D2-5

$\triangle \mathrm{D} 2-3$

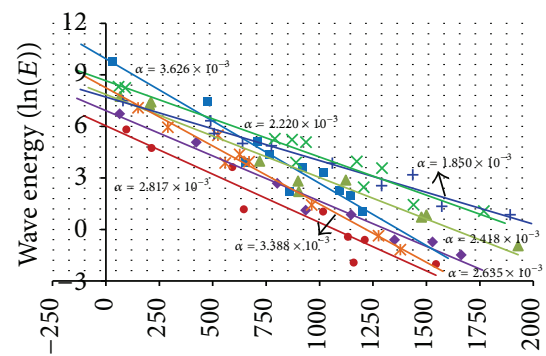

Wave propagation distance $(\mathrm{m})$

$\begin{array}{ll}\text { - D5-1 } & * \mathrm{D} 5-5 \\ \text { - D5-2 } & +\mathrm{D} 5-6 \\ \triangle \mathrm{D} 5-3 & \times \mathrm{D} 5-7 \\ \text { - D5-4 } & \end{array}$

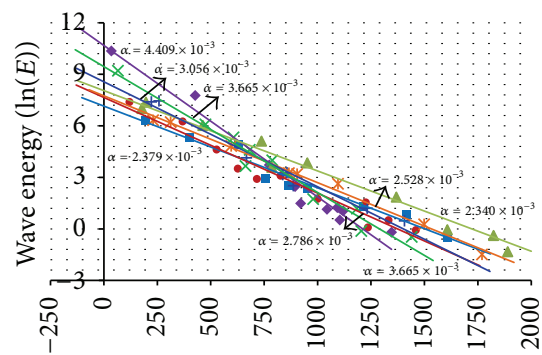

Wave propagation distance $(\mathrm{m})$
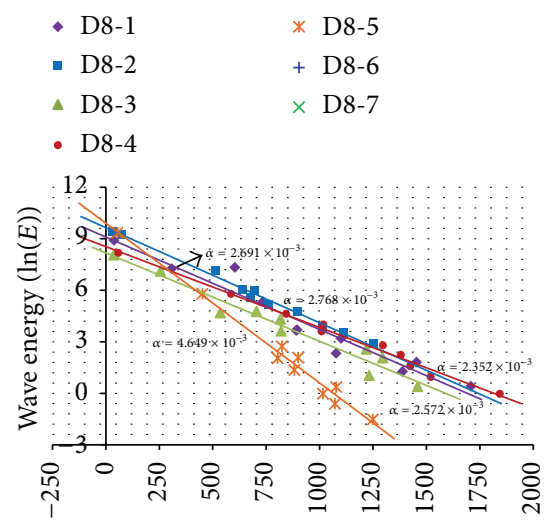

Wave propagation distance $(\mathrm{m})$

- D11-1

- D11-4

- D11-2

$\triangle$ D11-3

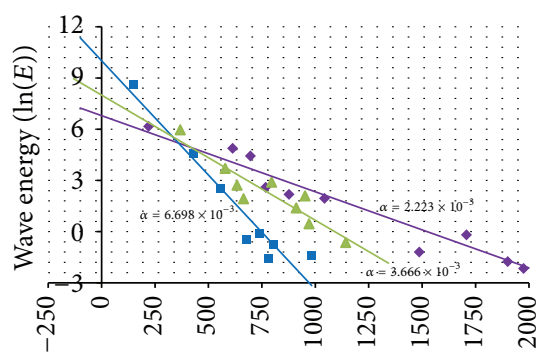

Wave propagation distance $(\mathrm{m})$

- D3-1

- D3-2

$\triangle \mathrm{D} 3-3$

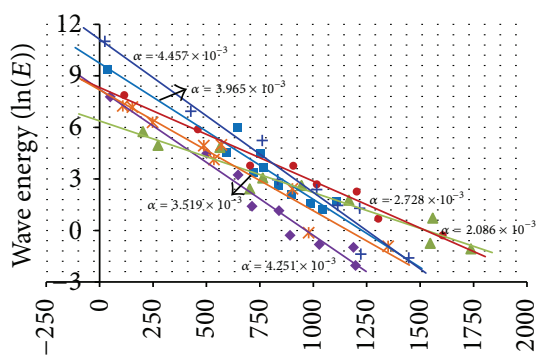

Wave propagation distance $(\mathrm{m})$

$\begin{array}{ll}\text { - D6-1 } & \text { - D6-4 } \\ \text { - D6-2 } & * \mathrm{D} 6-5 \\ \Delta \mathrm{D} 6-3 & +\mathrm{D} 6-6\end{array}$

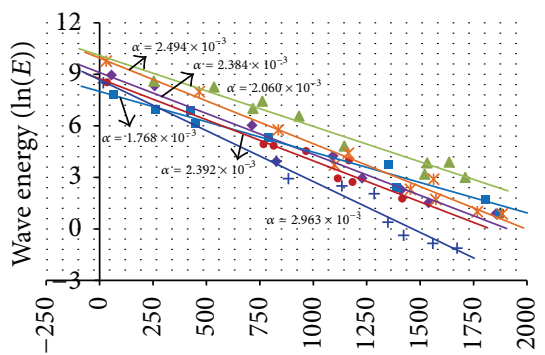

Wave propagation distance $(\mathrm{m})$

$\begin{array}{ll}\text { - D9-1 } & * \mathrm{D} 9-4 \\ \text { - } \mathrm{D} 9-2 & * \mathrm{D} 9-5 \\ \Delta \mathrm{D} 9-3 & +\mathrm{D} 9-6\end{array}$

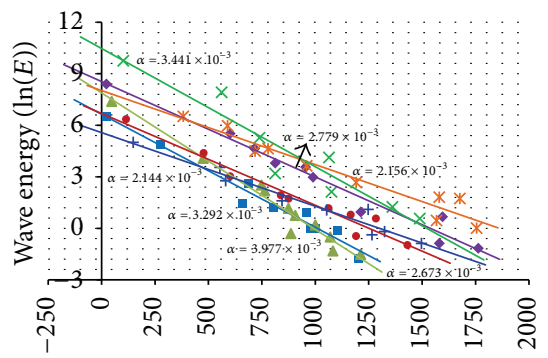

Wave propagation distance $(\mathrm{m})$

- D12-1 * D12-5

- D12-2 + D12-6

$\triangle \mathrm{D} 12-3 \times \mathrm{D} 12-7$

- D12-4

Figure 6: Continued. 

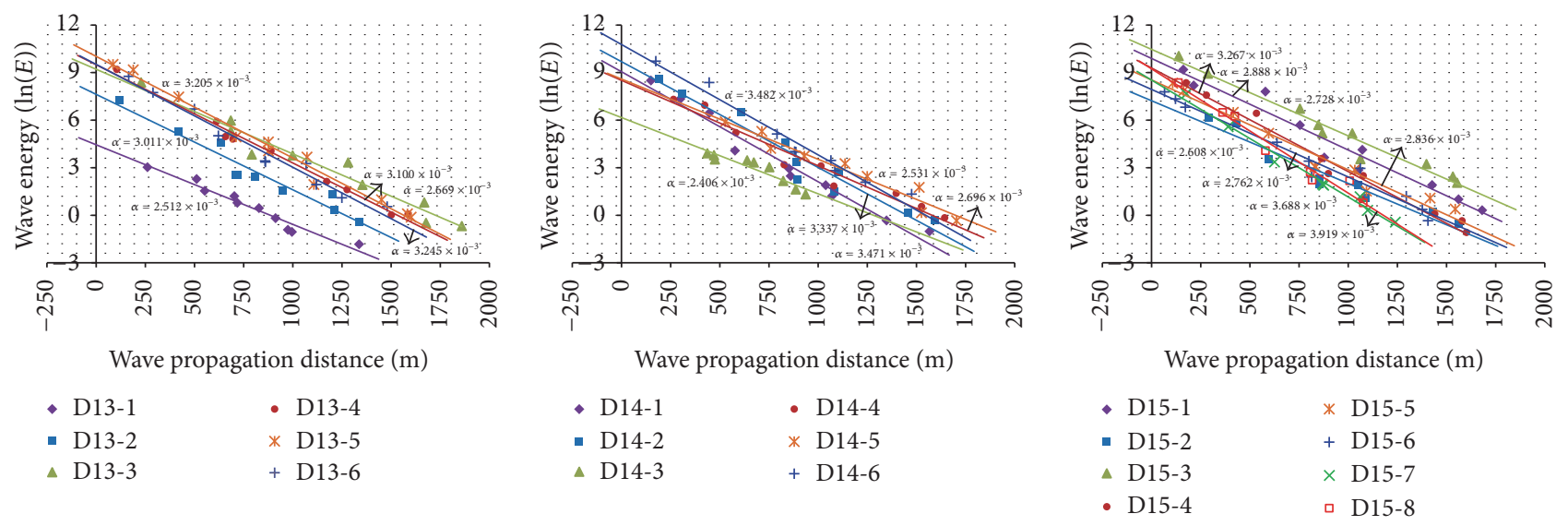

(b)

FIGURE 6: Linear relations of logarithmic residual wave energy and wave propagation distance for the great sample microseisms.

approach of solution algorithm used for figuring out the magnitude of focus energy releasing from a microseismic event can be summed up as follows: (1) effective components extraction of original microseismic wave; (2) focus repositioning upon reconstructed wave to obtain reliable wave propagation distance; (3) residual wave energy calculation; (4) fitting relationship establishment between wave propagation distance and residual wave energy; (5) obtaining the exponent $B$ and calculating focus energy.

5.2. Reliability. Microseismic focus energy determination is executed upon the real values of calibrated parameters. This fitting criterion attempts to obtain the focus energy as accurate as possible, which undoubtedly is an innovative method for exploring focus energy of unknown microseism and correcting the calculated microseismic focus energy. Compared to the practical algorithm using the wave energy attenuation, microseismic focus energy calculated by the energy density method is the average result of a number of probable focus energy values deduced from the microseismic waves on individual detectors. Average error gets greater with the increase of the amount of individual focus energy. And the dispersion of the individual focus energy for the same microseismic event also increases, which results in serious indeterminacy of the average microseismic focus energy. In contrast, microseismic focus energy determined by the new method is the optimal fitting value of all effective individuals, and the fitting result is unique. The fitting effectiveness in the linear relationship mirrors the accuracy of microseismic focus energy calculated by the new method. Indeterminacy of focus energy decreases with the restraint of multiple available residual wave energy individuals, and the standard error of the actual focus energy and fitting one decreases. In addition, two individual residual waves are basically satisfied for preliminarily estimating the focus energy when using the energy fitting determination. Yet, at least four individual residual waves are feasible to achieve the focus energy estimation in the energy density method. Advantages induced from the improved algorithm indicate that the reliability and accuracy of the microseismic focus energy resulting from the wave energy attenuation relationship are higher.

5.3. Uncertainty of the Determination of Microseismic Focus Energy. Like the previous methods, the accuracy of the microseismic focus energy computation based on the wave energy attenuation characteristics is closely correlated with the physical properties of rock medium including rock homogeneity. The higher the homogeneity of the rock medium is, the greater the accuracy of the microseismic focus energy is. The impact of geological structures, such as the faults, folds, and karst collapses, weakens rock homogeneity, especially in the deep mining circumstances. Roadways, goafs, and chambers also have an adverse impact on the rock homogeneity and result in the irregular microseismic wave energy attenuation. As such, spherical microseismic waves propagating in deep rock are not equiphase as the anisotropic features of rock medium. And the attenuation of residual microseismic wave energy gets more complicated for waves with longer propagation distance. Residual wave energy reflects the vibration intensity of rock particle induced by the microseismic event, which is greatly influenced by the geological structures, physical properties, and the types of rock medium. Rock property varies in different locations. It results in that the residual energy magnitude of microseismic sphere wave is quite different in separate directions and propagation paths and gets various for the same microseism, which to some extent brings adverse impact on the linear relationship between logarithmic residual wave energy and focus-to-detector distance. In particular, the linear relationship gets inconspicuous in the deep mining. This abnormal phenomenon in wave energy attenuation results in that the energy absorption coefficient as an average representation for rock properties changes easily. Thus, the accuracy and adaptability of the new method can be improved based upon the inversion of energy absorption coefficient in the complicated deep rock. A corrected parameter is generally necessary for an appropriate magnitude. Statistical features of these 92 energy absorption coefficients are shown in Figure 7. Energy absorption coefficient reflects the dissipation velocity 
TABLE 2: $B$ value and redetermined magnitude of the microseismic focus energy comparison.

\begin{tabular}{|c|c|c|c|c|}
\hline \multirow{2}{*}{ Wave group } & \multirow{2}{*}{$B$ value } & \multicolumn{2}{|c|}{ Microseismic focus energy/J } & \multirow{2}{*}{$\begin{array}{l}\text { Difference ratic } \\
\left(E_{R}-E_{O}\right) / E_{O}\end{array}$} \\
\hline & & Original & Redetermined & \\
\hline D1-1 & 7.967 & 1838.744 & 2883.627 & 0.568 \\
\hline D1-2 & 6.877 & 574.344 & 969.440 & 0.688 \\
\hline D1-3 & 7.688 & 889.335 & 2181.579 & 1.453 \\
\hline D1-4 & 7.804 & 875.636 & 2450.619 & 1.799 \\
\hline D1-5 & 12.217 & 96008.181 & 202294.736 & 1.107 \\
\hline D1-6 & 7.109 & 377.824 & 1223.027 & 2.237 \\
\hline D1-7 & 8.123 & 710.519 & 3371.011 & 3.744 \\
\hline D1-8 & 7.796 & 618.731 & 2431.934 & 2.931 \\
\hline D1-9 & 4.988 & 342.844 & 146.587 & -0.572 \\
\hline $\mathrm{D} 2-1$ & 10.277 & 2878.756 & 29070.058 & 9.098 \\
\hline $\mathrm{D} 2-2$ & 9.283 & 915.574 & 10754.483 & 10.746 \\
\hline D2-3 & 9.879 & 17571.647 & 19518.597 & 0.111 \\
\hline D2-4 & 9.379 & 993.190 & 11837.373 & 10.919 \\
\hline D2-5 & 8.237 & 929.051 & 3776.625 & 3.065 \\
\hline D3-1 & 6.794 & 975.155 & 892.733 & -0.085 \\
\hline D3-2 & 10.010 & 17069.975 & 22247.657 & 0.303 \\
\hline D3-3 & 7.997 & 858.596 & 2971.229 & 2.461 \\
\hline D4-1 & 8.461 & 351.830 & 4727.080 & 12.436 \\
\hline D4-2 & 8.532 & 280.423 & 5072.342 & 17.088 \\
\hline D4-3 & 9.804 & 8666.027 & 18109.664 & 1.090 \\
\hline D4-4 & 9.348 & 9094.919 & 11471.007 & 0.261 \\
\hline D5-1 & 6.917 & 798.185 & 1008.965 & 0.264 \\
\hline D5-2 & 9.936 & 7651.878 & 20667.341 & 1.701 \\
\hline D5-3 & 7.859 & 1534.007 & 2589.370 & 0.688 \\
\hline D5-4 & 6.035 & 81.631 & 417.778 & 4.118 \\
\hline D5-5 & 8.247 & 1756.953 & 3814.977 & 1.171 \\
\hline D5-6 & 7.710 & 1336.598 & 2230.250 & 0.669 \\
\hline D5-7 & 8.658 & 741.897 & 5754.538 & 6.757 \\
\hline D6-1 & 8.215 & 193.194 & 3694.587 & 18.124 \\
\hline D6-2 & 9.740 & 3721.416 & 16987.669 & 3.565 \\
\hline D6-3 & 6.377 & 1567.251 & 588.269 & -0.625 \\
\hline D6-4 & 8.323 & 342.728 & 4117.045 & 11.013 \\
\hline D6-5 & 8.184 & 703.436 & 3582.403 & 4.093 \\
\hline D6-6 & 11.128 & 878.651 & 68082.331 & 76.485 \\
\hline D7-1 & 8.695 & 3388.395 & 5975.679 & 0.764 \\
\hline D7-2 & 8.400 & 14896.719 & 4445.537 & -0.702 \\
\hline D7-3 & 7.982 & 862.068 & 2928.076 & 2.397 \\
\hline D7-4 & 8.278 & 312.303 & 3937.826 & 11.609 \\
\hline D7-5 & 8.475 & 649.662 & 4792.996 & 6.378 \\
\hline D7-6 & 7.605 & 806.959 & 2007.770 & 1.488 \\
\hline D7-7 & 8.563 & 2321.286 & 5235.272 & 1.255 \\
\hline D8-1 & 10.678 & 803.972 & 43393.459 & 52.974 \\
\hline D8-2 & 7.148 & 1608.375 & 1271.385 & -0.210 \\
\hline D8-3 & 8.049 & 5772.219 & 3131.906 & -0.457 \\
\hline D8-4 & 7.652 & 1821.967 & 2105.097 & 0.155 \\
\hline D8-5 & 7.748 & 3094.361 & 2317.034 & -0.251 \\
\hline D8-6 & 8.564 & 1790.451 & 5238.875 & 1.926 \\
\hline
\end{tabular}


TABLe 2: Continued.

\begin{tabular}{|c|c|c|c|c|}
\hline \multirow{2}{*}{ Wave group } & \multirow{2}{*}{$B$ value } & \multicolumn{2}{|c|}{ Microseismic focus energy/J } & \multirow{2}{*}{$\begin{array}{l}\text { Difference ratio } \\
\left(E_{R}-E_{O}\right) / E_{O}\end{array}$} \\
\hline & & Original & Redetermined & \\
\hline D8-7 & 9.448 & 2473.380 & 12682.914 & 4.128 \\
\hline D9-1 & 9.103 & 3843.190 & 8981.166 & 1.337 \\
\hline D9-2 & 8.003 & 33776.282 & 2991.000 & -0.911 \\
\hline D9-3 & 10.094 & 98254.202 & 24200.631 & -0.754 \\
\hline D9-4 & 8.744 & 4258.074 & 6272.436 & 0.473 \\
\hline D9-5 & 9.945 & 6926.762 & 20853.248 & 2.011 \\
\hline D9-6 & 8.680 & 844.062 & 5885.995 & 5.973 \\
\hline D10-1 & 7.731 & 1713.440 & 2278.872 & 0.330 \\
\hline D10-2 & 6.670 & 671.156 & 788.251 & 0.174 \\
\hline D10-3 & 8.007 & 647.045 & 3003.120 & 3.641 \\
\hline D10-4 & 7.708 & 396.921 & 2225.874 & 4.608 \\
\hline D10-5 & 8.315 & 2069.528 & 4083.713 & 0.973 \\
\hline D11-1 & 9.099 & 3261.223 & 8946.530 & 1.743 \\
\hline D11-2 & 9.644 & 15963.736 & 15426.545 & -0.034 \\
\hline D11-3 & 8.173 & 1721.555 & 3545.027 & 1.059 \\
\hline D11-4 & 8.540 & 4517.461 & 5115.436 & 0.132 \\
\hline D11-5 & 9.902 & 909.451 & 19962.544 & 20.950 \\
\hline D12-1 & 8.546 & 1730.590 & 5144.379 & 1.973 \\
\hline D12-2 & 6.641 & 187.526 & 765.665 & 3.083 \\
\hline D12-3 & 7.886 & 434.279 & 2659.400 & 5.124 \\
\hline D12-4 & 6.687 & 265.001 & 802.132 & 2.027 \\
\hline D12-5 & 8.023 & 12161.523 & 3050.135 & -0.749 \\
\hline D12-6 & 5.572 & 325.080 & 262.838 & -0.191 \\
\hline D12-7 & 10.470 & 3485.852 & 35229.814 & 9.107 \\
\hline D13-1 & 4.447 & 229.590 & 85.399 & -0.628 \\
\hline D13-2 & 7.628 & 918.990 & 2055.498 & 1.237 \\
\hline D13-3 & 9.213 & 9196.919 & 10021.688 & 0.090 \\
\hline D13-4 & 9.500 & 10776.991 & 13356.470 & 0.239 \\
\hline D13-5 & 10.025 & 5122.511 & 22575.164 & 3.407 \\
\hline D13-6 & 9.519 & 11364.339 & 13612.694 & 0.198 \\
\hline D14-1 & 9.057 & 726.592 & 8582.068 & 10.811 \\
\hline D14-2 & 9.692 & 2518.948 & 16193.715 & 5.429 \\
\hline D14-3 & 6.165 & 1332.728 & 475.631 & -0.643 \\
\hline D14-4 & 8.515 & 2896.582 & 4989.234 & 0.722 \\
\hline D14-5 & 8.597 & 3470.861 & 5413.433 & 0.560 \\
\hline D14-6 & 10.775 & 4377.518 & 47792.979 & 9.918 \\
\hline D15-1 & 9.894 & 5197.978 & 19812.785 & 2.812 \\
\hline D15-2 & 7.226 & 593.022 & 1375.085 & 1.319 \\
\hline D15-3 & 10.467 & 18903.281 & 35142.490 & 0.859 \\
\hline D15-4 & 9.292 & 3013.907 & 10852.587 & 2.601 \\
\hline D15-5 & 8.540 & 2600.196 & 5116.402 & 0.968 \\
\hline D15-6 & 7.939 & 2005.059 & 2805.073 & 0.399 \\
\hline D15-7 & 8.530 & 411.417 & 5064.845 & 11.311 \\
\hline D15-8 & 9.238 & 1360.940 & 10279.781 & 6.553 \\
\hline
\end{tabular}




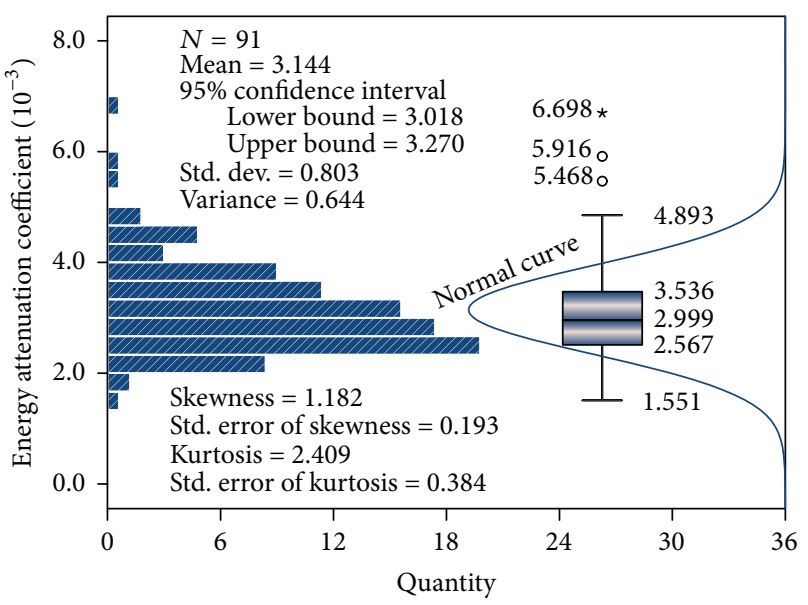

Figure 7: Histogram, boxplot, normal test, and Q-Q plot of observed EAC magnitude.

of the focus energy in rock medium. The magnitude is closely related to the physical properties of rock. Energy absorption coefficient provides an indication of how effective a given rock medium is in promoting energy interactions. Individual energy absorption coefficient is quite different in separate locations.

5.4. Impact of Built-In Velocity Threshold. In addition, due to the influence of built-in velocity threshold of the monitoring firmware, it is always found that the waveform of a few of microseismic events is incomplete as shown in Figure 8. This causes that components of dominant particle velocity which exceed the threshold are unable to be recorded. This data deficiency gets more serious as the focus-to-detector distance is closer, especially for the events with the serious releasing elastic energy. These missed dominant wave components are unable to be retrieved. This results in that the calculated residual wave energy is obviously less than the actual energy the wave carries, which brings adverse impact on the accuracy. Such phenomena are shown in Figures 8 and 9.

Wave energy distribution in frequency spectrum changes with the velocity threshold. Wave energy loss induced by the velocity threshold mainly appears in the low frequency range, located around the basic frequency. Meanwhile, the restricted wave energy increases linearly with the growth of velocity threshold. It indicates that although those lost dominant wave components are unable to be fixed, the linear relationship between the velocity threshold and residual energy implies the relative reliability of microseismic wave energy prediction once a probable PPV is given. Based on this linear relation and probable PPV confirmed by the trend curve, the missed microseismic wave energy at a near-source location is able to recover to a certain degree. The accuracy of the focus energy can then be corrected. It will greatly increase the utilization efficiency of impaired microseismic wave data. Only when the waveform integrity is ensured can residual wave energy be possibly abstracted accurately. The new determination method for microseismic focus energy has high dependence on the integrity of waveform, accuracy of focus position, and reliability of effective wave components.

\section{Conclusions}

(1) In view of the seismic energy attenuation characteristics in deep rock, a mathematical solution for determining the focus energies of microseismic events was proposed. In the solution, improved wave propagation distances and residual wave energies are the two critical and fundamental factors for seismic energy determination. The propagation distances of the seismic waves are more accurately calculated using focus repositioning, and the equivalent residual energies of seismic waves were confirmed using the wavelet packet transform. After the relevant Napierian logarithmic transformation, the linear attenuation relationship between the wave propagation distance and the logarithmic residual seismic energy was established on the basis of the seismic wave attenuation characteristics. The seismic focus energy was then inversely determined by the intercept of the fitted straight line.

(2) The calculated microseismic focus energy is the optimal fitting magnitude resulting from the individual residual wave energies. Given the constraints of multiple available residual wave energy individuals, the standard deviation between the actual focus energy and the fitting energy greatly decreases. In addition, the uniqueness of the energy computation approach increases as the number of available residual individuals increases. In the improved algorithm, the seismic energy determination is executed using the real values of the calibrated parameters. The solution is highly dependent on the integrity of the seismic waveforms, the accuracy of the focus position, and the effectiveness of the wave components. Waveform restoration increases the utilization efficiency of impaired seismic wave records. The reliability of the determined seismic energy is therefore greatly improved.

(3) Given the anisotropic features of a rock medium, seismic waves change with directions and propagation paths. Energy attenuation becomes more complicated for residual waves with longer focus-to-detector distances. Seismic energy determination using residual wave attenuation characteristics is greatly influenced by rock properties and structures. The seismic energy reliability increases with increasing rock homogeneity. The energy absorption coefficient is a significant representation of the seismic energy dissipation in the propagation medium, which is sensitive to the rock properties and structures. The reliability and adaptability of the seismic energy determination in complicated rock are improved using the inversion of the energy absorption coefficient.

(4) It must be noted that the proposed microseismic focus energy computation method in this research is a key fundamental framework, and further studies will be undertaken mainly around two points: (1) the investigation on the logical algorithm between total energy released from the microseismic event and initial energy used for propagating in rock medium in the form of stress wave; (2) the exploration on the correction coefficient of the computation method to improve its adaptability in rough geological environments. 


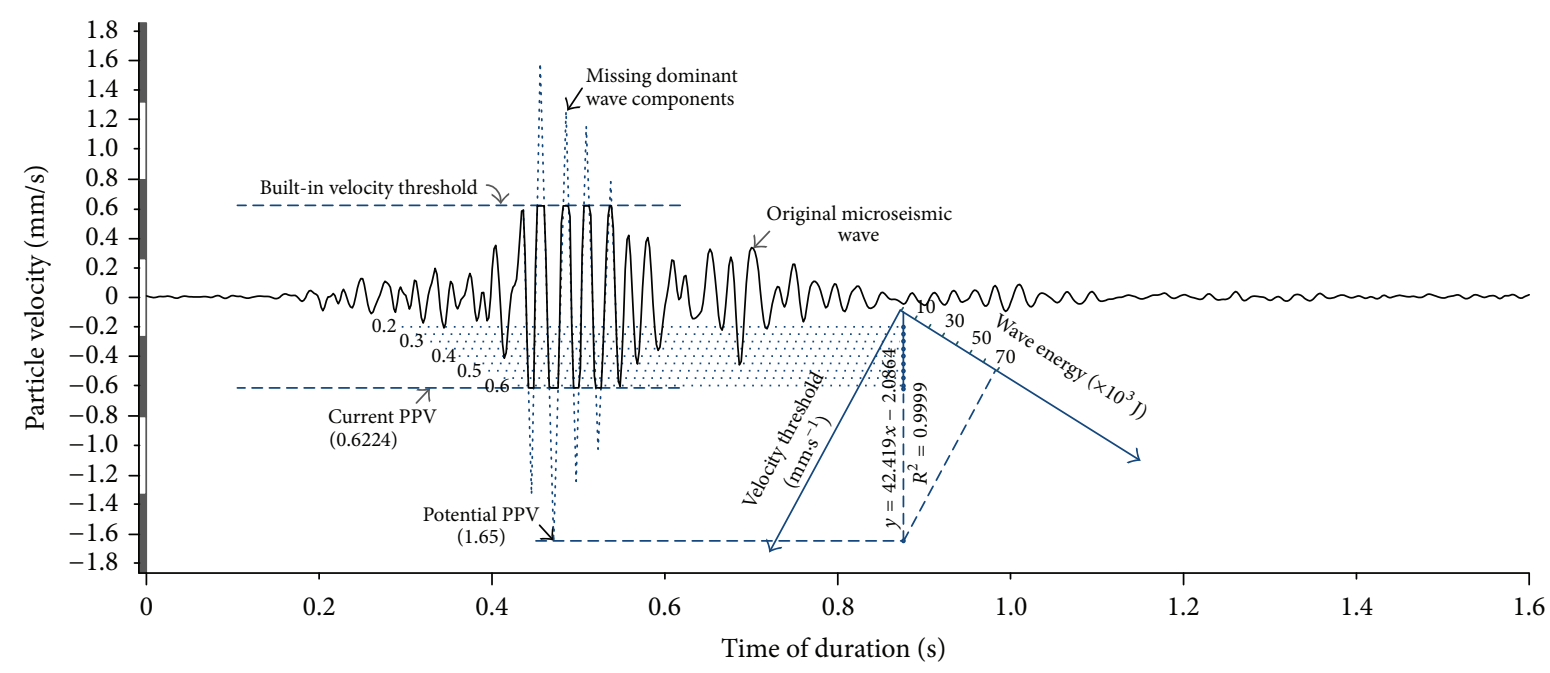

FIGURE 8: Incomplete measured waveform of the high-energy microseismic event and the potential relationship between residual wave energy and velocity threshold.

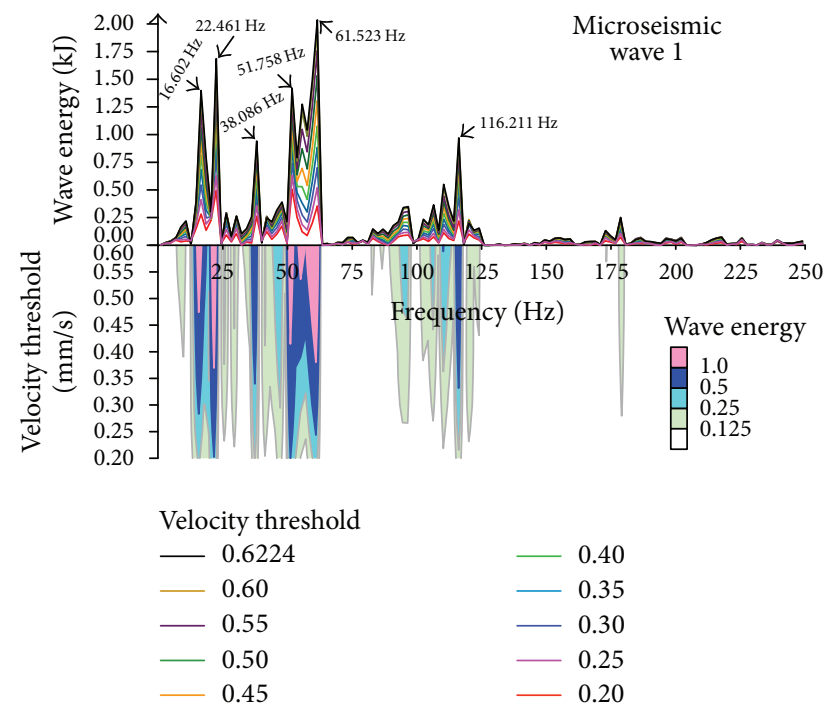

FIGURE 9: Changes of wave energy in frequency range with the increase of velocity threshold.

\section{Conflicts of Interest}

The authors declare that there are no conflicts of interest regarding the publication of this paper.

\section{Acknowledgments}

The financial and general support for this research provided by the Fundamental Research Funds for the Central Universities (2015QNA62) and the National Key R\&D Program of China (no. 2016YFC0600900) is gratefully acknowledged. Additionally, the authors express their sincere gratitude to the Xingcun Coal Mine for its support of the microseismic field experiment.

\section{References}

[1] Y. Chen, Y. Hao, Y. Wu, K. Zhang, and G. Zhang, “The load capacity model and experimental tests of a new yielding steel prop," Mathematical Problems in Engineering, vol. 2017, 11 pages, 2017.

[2] M. Zhang, H. Shimada, T. Sasaoka, K. Matsui, and L. Dou, "Seismic energy distribution and hazard assessment in underground coal mines using statistical energy analysis," International Journal of Rock Mechanics and Mining Sciences, vol. 64, pp. 192-200, 2013.

[3] Y. Chen, A. Lu, X. Mao, M. Li, and L. Zhang, "Nonlinear dynamics mechanism of rock burst induced by the instability of the layer-crack plate structure in the coal wall in deep coal mining," Shock and Vibration, vol. 2017, pp. 1-12, 2017.

[4] L. Dong, J. Wesseloo, Y. Potvin, and X. Li, "Discrimination of mine seismic events and blasts using the fisher classifier, naive bayesian classifier and logistic regression," Rock Mechanics and Rock Engineering, vol. 49, no. 1, pp. 183-211, 2016.

[5] A. Leśniak and Z. Isakow, "Space-time clustering of seismic events and hazard assessment in the Zabrze-Bielszowice coal mine, Poland," International Journal of Rock Mechanics and Mining Sciences, vol. 46, no. 5, pp. 918-928, 2009.

[6] L.-J. Dong, J. Wesseloo, Y. Potvin, and X.-B. Li, "Discriminant models of blasts and seismic events in mine seismology," International Journal of Rock Mechanics \& Mining Sciences, vol. 86, pp. 282-291, 2016.

[7] C. R. Froese, F. Moreno, M. Jaboyedoff, and D. M. Cruden, "25 years of movement monitoring on South Peak, Turtle Mountain: Understanding the hazard," Canadian Geotechnical Journal, vol. 46, no. 3, pp. 256-269, 2009.

[8] L. Dong, W. Shu, X. Li, G. Han, and W. Zou, “Three dimensional comprehensive analytical solutions for locating sources of sensor networks in unknown velocity mining system," IEEE Access, vol. 5, pp. 11337-11351, 2017.

[9] G.-A. Tselentis, N. Martakis, P. Paraskevopoulos, A. Lois, and E. Sokos, "Strategy for automated analysis of passive microseismic data based on S-transform, Otsu's thresholding, and higher order statistics," Geophysics, vol. 77, no. 6, pp. KS43-KS54, 2012. 
[10] M. Zhang, H. Shimada, T. Sasaoka, K. Matsui, and L. Dou, "Evolution and effect of the stress concentration and rock failure in the deep multi-seam coal mining," Environmental Earth Sciences, vol. 72, no. 3, pp. 629-643, 2014.

[11] A. Kushnir, A. Varypaev, I. Dricker, M. Rozhkov, and N. Rozhkov, "Passive surface microseismic monitoring as a statistical problem: Location of weak microseismic signals in the presence of strongly correlated noise," Geophysical Prospecting, vol. 62, no. 4, pp. 819-833, 2014.

[12] O. Zhebel and L. Eisner, "Simultaneous microseismic event localization and source mechanism determination," Geophysics, vol. 80, no. 1, pp. KS1-KS9, 2015.

[13] H.-X. Miao, F.-X. Jiang, X.-J. Song, J.-Y. Song, S.-H. Yang, and J.-R. Jiao, "Automatically positioning microseismic sources in mining by the stereo tomographic method using full wavefields," Applied Geophysics, vol. 9, no. 2, pp. 168-176, 2012.

[14] A. Venkataraman and H. Kanamori, "Effect of directivity on estimates of a radiated seismic energy," Journal of Geophysical Research: Solid Earth, vol. 109, no. 4, pp. B04301-12, 2004.

[15] J. Shi, P. G. Richards, and W.-Y. Kim, "Determination of seismic energy from Lg waves," Bulletin of the Seismological Society of America, vol. 90, no. 2, pp. 483-493, 2000.

[16] A. Hofstetter and A. Shapira, "Determination of earthquake energy release in the Eastern Mediterranean region," Geophysical Journal International, vol. 143, no. 3, pp. 898-908, 2000.

[17] D. Choudhury and A. D. Katdare, "New approach to determine seismic passive resistance on retaining walls considering seismic waves," International Journal of Geomechanics, vol. 13, no. 6, pp. 852-860, 2013.

[18] J. A. Sanchidrian, P. Segarra, and L. M. Lopez, "Energy components in rock blasting," International Journal of Rock Mechanics and Mining Sciences, vol. 44, no. 1, pp. 130-147, 2007.

[19] H.-X. Miao, F.-X. Jiang, X.-J. Song, S.-H. Yang, and J.-R. Jiao, "Tomographic inversion for microseismic source parameters in mining," Applied Geophysics, vol. 9, no. 3, pp. 341-348, 2012.

[20] D. Rhodes, "Energy density," Scientific American, vol. 304, no. 2, p. 8, 2011.

[21] R. G. Kulinich, M. G. Valitov, and Z. N. Proshkina, "A comparative analysis of the seismic and density models for the Earth's Crust of the Central Kurils," Russian Journal of Pacific Geology, vol. 9, no. 6, pp. 439-450, 2015.

[22] A. F. C. Errington, B. L. F. Daku, A. F. Prugger, and D. E. Dodds, "Energy spectral density characterization of microseismic events in potash mines," Measurement, vol. 42, no. 2, pp. 264-268, 2009.

[23] K. K. F. Wong, "Inelastic seismic response analysis based on energy density spectra," Journal of Earthquake Engineering, vol. 8, no. 2, pp. 315-334, 2004.

[24] J.-P. Liu, X.-T. Feng, Y.-H. Li, S.-D. Xu, and Y. Sheng, "Studies on temporal and spatial variation of microseismic activities in a deep metal mine," International Journal of Rock Mechanics and Mining Sciences, vol. 60, pp. 171-179, 2013.

[25] S. Sasaki and H. Kaieda, "Determination of stress state from focal mechanisms of microseismic events induced during hydraulic injection at the Hijiori hot dry rock site," Pure and Applied Geophysics, vol. 159, no. 1-3, pp. 489-516, 2002.

[26] M. Hudyma and Y. H. Potvin, "An engineering approach to seismic risk management in hardrock mines," Rock Mechanics and Rock Engineering, vol. 43, no. 6, pp. 891-906, 2010.

[27] M. Cai, P. K. Kaiser, and C. D. Martin, "Quantification of rock mass damage in underground excavations from microseismic event monitoring," International Journal of Rock Mechanics and Mining Sciences, vol. 38, no. 8, pp. 1135-1145, 2001.

[28] A. J. Feustel, "Seismic attenuation in underground mines: A comparative evaluation of methods and results," Tectonophysics, vol. 289, no. 1-3, pp. 31-49, 1998.

[29] P. N. Adams, R. S. Anderson, and J. Revenaugh, "Microseismic measurement of wave-energy delivery to a rocky coast," Geology, vol. 30, no. 10, pp. 895-898, 2002.

[30] J. M. Carcione, D. Kosloff, and R. Kosloff, "Wave propagation simulation in a linear viscoelastic medium," Geophysical Journal International, vol. 95, no. 3, pp. 597-611, 1988.

[31] T. L. Chelidze, H. A. Spetzler, and G. A. Sobolev, "Absorption of strain waves in porous media at seismic frequencies," Pure and Applied Geophysics, vol. 147, no. 1, pp. 25-55, 1996.

[32] B. L. Kennett, Seismic Wave Propagation in Stratified Media, ANU E Press, 2009.

[33] J. Pujol and S. Smithson, "Seismic wave attenuation in volcanic rocks from VSP experiments," Geophysics, vol. 56, no. 9, pp. 1441-1455, 1991.

[34] G.-L. Feng, X.-T. Feng, B.-R. Chen, and Y.-X. Xiao, "Performance and feasibility analysis of two microseismic location methods used in tunnel engineering," Tunnelling and Underground Space Technology, vol. 63, pp. 183-193, 2017.

[35] H. Toda, Z. Zhang, and T. Imamura, "The design of complex wavelet packet transforms based on perfect translation invariance theorems," International Journal of Wavelets, Multiresolution and Information Processing, vol. 8, no. 4, pp. 537-558, 2010.

[36] Z. Prusa, P. L. Søndergaard, and P. Rajmic, "Discrete wavelet transforms in the large time-frequency analysis toolbox for MATLAB/GNU octave," ACM Transactions on Mathematical Software, vol. 42, no. 4, article no. 32, 2016.

[37] M. Zhang, T. Sasaoka, H. Shimada, and K. Matsui, "Comparison of optimal basis function for the underground microseismic wave processing in wavelet packet transform," Memoirs of the Faculty of Engineering, Kyushu University, vol. 73, no. 3, pp. 7185, 2013. 


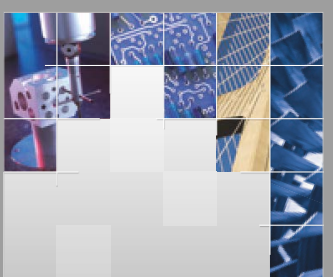

\section{Enfincering}
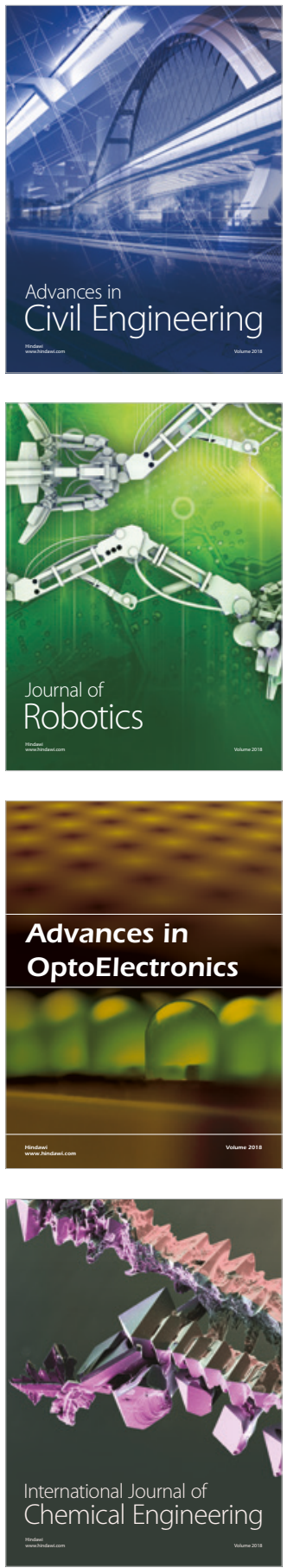

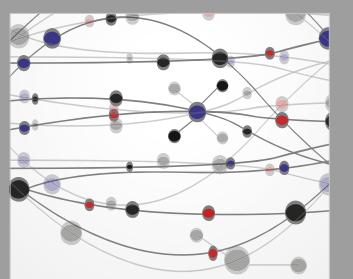

\section{Rotating \\ Machinery}

The Scientific World Journal

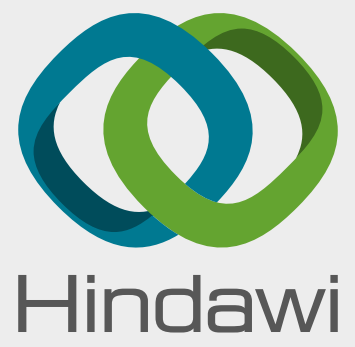

Submit your manuscripts at

www.hindawi.com
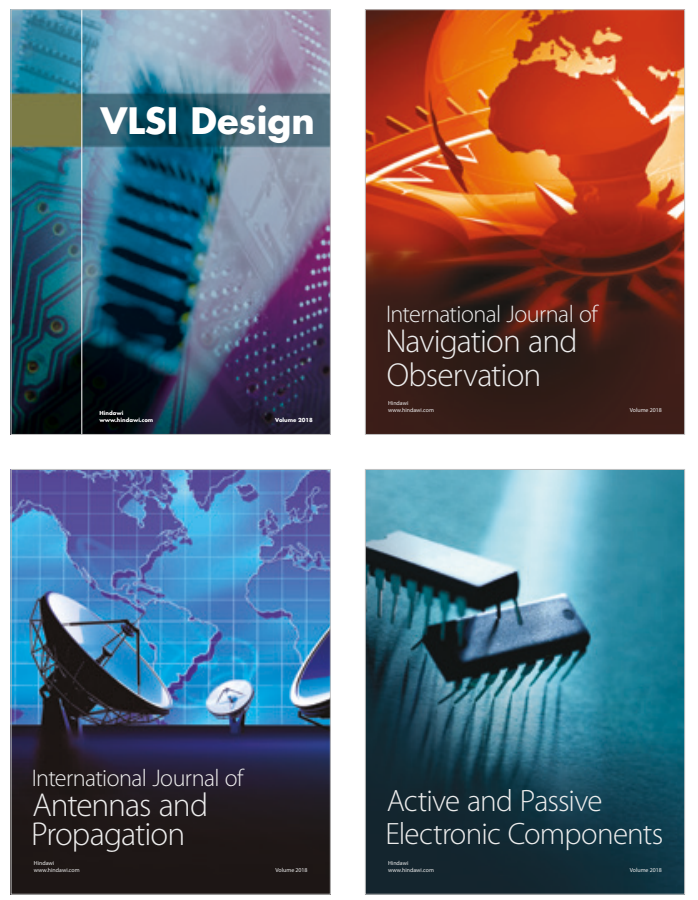
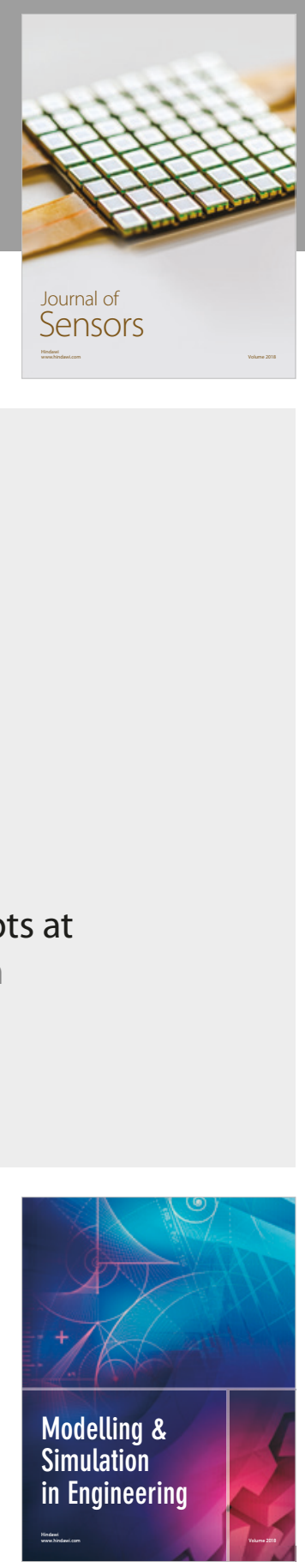

\section{Advances \\ Multimedia}
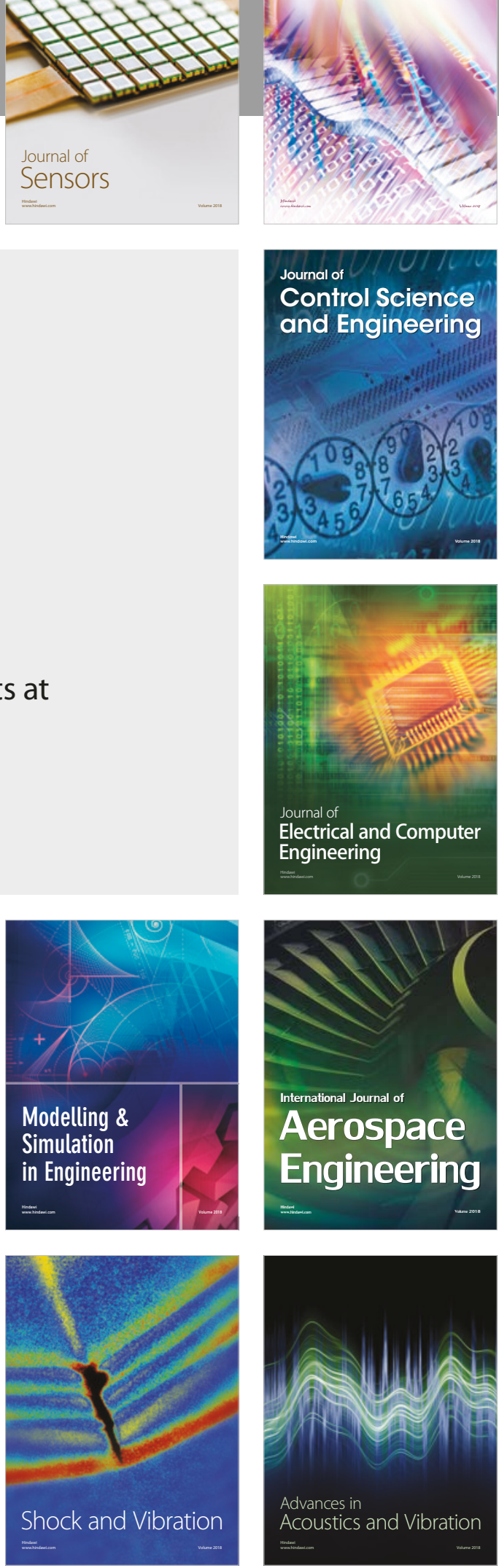